\title{
The time course of ethanol tolerance: associative learning
}

J.L.O. Bueno and A. Fachini
Setor de Psicobiologia, Departamento de Psicologia e Educação, Faculdade de Filosofia, Ciências e Letras de Ribeirão Preto, Universidade de São Paulo, Ribeirão Preto, SP, Brasil

\section{Correspondence}

J.L.O. Bueno

Departamento de Psicologia e

Educação, FFCLRP, USP

Av. Bandeirantes, 3900

14040-901 Ribeirão Preto, SP

Brasil

E-mail: jldobuen@ffclrp.usp.br

Publication supported by FAPESP. ..........................

Received October 23, 2006 Accepted July 27, 2007

\begin{abstract}
The effect of different contextual stimuli on different ethanol-induced internal states was investigated during the time course of both the hypothermic effect of the drug and of drug tolerance. Minimitters were surgically implanted in 16 Wistar rats to assess changes in their body temperature under the effect of ethanol. Rat groups were submitted to ethanol or saline trials every other day. The animals were divided into two groups, one receiving a constant dose (CD) of ethanol injected intraperitoneally, and the other receiving increasing doses (ID) during the 10 training sessions. During the ethanol training sessions, conditioned stimuli A (tone) and B (buzzer) were presented at "state +" (35 min after drug injection) and "state -" (170 min after drug injection), respectively. Conditioned stimuli C (bip) and D (white noise) were presented at moments equivalent to stimuli $\mathrm{A}$ and $\mathrm{B}$, respectively, but during the saline training sessions. All stimuli lasted 15 min. The CD group, but not the ID group, developed tolerance to the hypothermic effect of ethanol. Stimulus A (associated with drug "state +") induced hyperthermia with saline injection in the ID group. Stimulus B (associated with drug "state -") reduced ethanol tolerance in the CD group and modulated the hypothermic effect of the drug in the ID group. These results indicate that contextual stimuli acquire modulatory conditioned properties that are associated with the time course of both the action of the drug and the development of drug tolerance.
\end{abstract}

Key words

- Ethanol tolerance

- Associative learning

- Time course

- Context stimuli

- Internal states

.....................

\section{Introduction}

Drug tolerance is a process characterized by the progressive decrease in the effect of a drug on an organism over the course of repeated administrations at the same dose and concentration (1). For example, the fourth injection of ethanol in rats induces less hypothermia than the first $(2,3)$. This decreasing effect of a drug following each successive administration is termed chronic tolerance. The term 'tolerance' is widely used to refer to such chronic tolerance.
Most investigators (e.g., Ref. 4) emphasize the contribution of neurochemical and physiological factors to the development of tolerance. However, learning also has been considered to be a central process in the development of tolerance (5-8).

Many experimental findings have demonstrated that tolerance to the hypothermic effect of ethanol is substantially reduced in rats when ethanol is administered in an environment with which the drug is not associated (9-11). This environmental specificity, indicated by loss of tolerance in a new con- 
text, emphasizes both the involvement of a learning process and contextual environmental dependence in the development of drug tolerance.

Siegel (12) has emphasized the contribution of drug-associated cues to tolerance in a Pavlovian conditioning analysis of drug tolerance. According to the usual conditioning terminology, the primary effect of a drug constitutes the unconditioned stimulus. Prior to any learning, this pharmacological stimulation elicits physiological responses in the organism that compensate for the primary drug-induced effects (6) and are known as unconditioned responses. Cues presented at the time of drug administration operate as conditioned stimuli. After a few pairings of the pre-drug conditioned stimuli (cues) and pharmacological unconditioned stimuli (drug), a drug-compensatory response is elicited as a conditioned response. The conditioned compensatory responses mediate the development of tolerance by counteracting the effect of the drug. For example, in rats that become tolerant to the hypothermic effect of ethanol, administration of an inert substance in the presence of ethanol-associated cues results in hyperthermia (13). This hyperthermia is a conditioned compensatory response that attenuates the thermal effect of ethanol when administered in the presence of drug-paired stimuli. Thus, through a learning process, such drug-associated cues acquire the property of anticipating the homeostatic pharmacologically induced imbalance, eliciting a homeostatic counteradjustment response to pharmacological stimulation.

Studies of drug tolerance have used a procedure of conditioned stimuli associated with the effect of ethanol, but the time course of the action of the drug has not been considered. Recognizing the development of different states during the time course of a drug effect (14) implies that the strength of association of the exteroceptive contextual stimuli with the drug effect may differ, depending on the time point of the animal's current state. For example, Elsmore and Manning (15) manipulated the time parameters of delta-9-THC administration before training sessions in rats submitted to interval schedules of food reinforcement, showing that the magnitude and direction of the drug effects on the animal's performance depends on these time parameters.

Many studies of learned associations between internal states induced by different drugs contemplate the role of temporal parameters in the administration of two drugs. For example, Revusky and Coombes (16) examined the associative consequences of the temporal relationship between a pentobarbital injection and a subsequent lithium injection. After forward pairing (pentobarbital before lithium), with delays between the two injections ranging from 2.5 to 320 min among groups, elimination or attenuation of the pentobarbital-produced aversion to saccharin was observed. Analogous to this procedure, these investigators suggest the use of a tone of 320-min duration as a conditioning procedure before a shock. To simulate the pentobarbital state, the tone should reach its maximum intensity after an hour at most and then gradually decrease, becoming very weak at the time of the shock. According to Revusky and Coombes (16), no experienced student of animal learning would expect to observe conditioning to such a tone since, for most of the 320-min duration of the procedure, the tone would be related to the absence of the unconditioned stimulus, and only the final, weak intensity tone would be related to the shock.

There is evidence that associations between two internal states (such as those produced by Revusky and Coombes (16)) may contribute to tolerance (17). For example, Taukulis (18) described the results of an experiment in which atropine sulfate was routinely injected prior to pentobarbital. Tolerance to the hypothermic effect of barbiturate was much more pronounced when 
atropine was injected first than in the absence of this anti-cholinergic signal. As discussed by Siegel (19), such pharmacological associations may manifest themselves as state-dependent-learning tolerance.

State-dependent-learning experiments show that subjects trained to perform a task under the effect of certain drugs often display a failure in their learning performance when tested in the absence of such drugs (20). However, this failure is reduced when the drugs are reintroduced. In this case, both the changes in the internal state produced by changes in the exteroceptive sensory context and the state-dependent-learning phenomenon can be viewed as instances of contextual stimulus control (21). The state-dependent-learning process has been investigated using ethanol-produced effects $(22,23)$ and morphine tolerance development (19).

These studies emphasize either drug-produced, interoceptive cue association with behavior performance (such as state-dependent-learning) or a different interoceptive cue by drugs (such as drug-drug associations). However, exteroceptive stimuli may be associated with a central motivational state that promotes the organization of consummatory, instrumental and humoral components of the behavioral repertoire $(24,25)$. There is no clear understanding of the relationship between exteroceptive stimuli and internal states associated with the time course of both maximum drug action and the evanescence or loss of drug effect. Thus, our objective in the present investigation was to examine the development of drug tolerance and to determine how exteroceptive contextual stimuli are associated with both the time course of drug action and drug tolerance.

In summary, to provide exteroceptive control of internal states, auditory stimuli will be presented during two different moments of the time course of drug action: the maximum and the evanescence effects. The hypothermic effect of ethanol will be controlled during the tolerance process along the sessions through constant maintenance of ethanol dose in one group and the increasing of ethanol dose in another group. Test sessions after the training will examine the properties of the exteroceptive contextual stimuli associated with the internal states in the time course of drug action and drug tolerance.

\section{Material and Methods}

\section{Animals}

The subjects were 24 male Wistar rats, experimentally naive, bred in the Central Colony of the University of São Paulo at Ribeirão Preto. The rats were about 90 days old at the beginning of the experiment, weighed 300 to $330 \mathrm{~g}$, and had free access to food and water. The animals were housed singly in plastic cages in the laboratory colony room, at $24 \pm 0.5^{\circ} \mathrm{C}$, on a 12-h light:dark cycle (lights on from 6:00 to 18:00). Eight rats were assigned to a group receiving ethanol at a constant dose (CD) and 16 rats to a group receiving an increasing ethanol dose (ID).

\section{Apparatus and drug}

The tests were performed in an acoustically isolated experimental room at ambient temperature $\left(23.5 \pm 0.5^{\circ} \mathrm{C}\right)$. The room was illuminated by a dim red light. The rats were placed in individual, clear, plastic cages (30 x $38 \times 16 \mathrm{~cm}$ ), approximately $35 \mathrm{~cm}$ apart, in a semi-circular arrangement.

The body temperature of each animal was measured by an implanted biotelemetry device (minimitter or temperature transmitter; Mini-Mitter Company Inc., Sunriver, OR, USA, model VM-FH). The minimitter was encased in a cylindrical capsule, 19-mm long x $12 \mathrm{~mm}$ in diameter, weighing $2.3 \mathrm{~g}$, and was implanted into the peritoneal cavity under pentobarbital anesthesia (for details about the surgical procedure, see Ref. 17). 
The transmitter emits a radio frequency (RF) signal at a rate proportional to the rat's body temperature. This signal is picked up by RF receivers placed under each plastic cage and connected to a matrix (BCM100, Mini-Mitter Company Inc.), which was cable-connected to a computer in a neighboring control room where the registration process was monitored.

The RF signal was transformed to a temperature unit (accurate to $\pm 0.05^{\circ} \mathrm{C}$ ) by software installed in the computer (Dataquest III, Saint Paul, MN, USA). The system allows the collection of temperature data each minute simultaneously from up to 8 rats.

Four auditory stimuli were used as conditioning stimuli: a 1-kHz tone (A), a buzzer (B), a bip (C), and white noise (D). Stimuli were produced by a software (Csound, Cambridge, MA, USA) that generated a WAV-file for each auditory stimulus. These stimuli were recorded on 90-min EMTEC FEI tape using a Philips (São Paulo, SP, Brazil) sound system, model AW7520. A single speaker (27 W) for delivering the auditory stimuli was placed near the ceiling of the experimental room and connected to the sound system in the control room, where the stimuli were controlled.

The drug used was ethanol (100\%), which was always administered at a dose of $1.4 \mathrm{~g} /$ $\mathrm{kg}$ to the $\mathrm{CD}$ group. In order to maintain the hypothermic effect during the training sessions, the ID group received increasing ethanol doses in three steps: two injections of 1.2 $\mathrm{g} / \mathrm{kg}$ and six of $1.6 \mathrm{~g} / \mathrm{kg}$ and of $2.0 \mathrm{~g} / \mathrm{kg}$ during the subsequent sessions. Control saline injections were adinistered as $5 \mathrm{~mL} / \mathrm{kg}$, as were the ethanol injections. All ethanol and saline injections were administered intraperitoneally.

\section{Procedure}

Habituation and baseline. All rats were handled daily for 3 min for 7 days before surgical implantation of the temperaturetelemetry devices. The animals were allowed to recover for a further 7 days, being treated daily with rifocin spray before participating in the experiments. After recovery, the experiments were started using two habituation sessions. The rats were taken from their home cages to the experimental room for $1 \mathrm{~h}$ on the first day and for $2 \mathrm{~h}$ on the second day. No stimulus was presented during this phase. Following habituation, five baseline sessions were run daily. The animals were taken to the experimental room and confined in a temperature-measurement cage for $1 \mathrm{~h}$.

Training sessions. During the training phase, both CD and ID rats received 10 ethanol and 10 saline injections in an alternating drug schedule, one injection every other day. Injections were given $20 \mathrm{~min}$ after transfer to the experimental room over a period of approximately $5 \mathrm{~min}$. The rats were injected sequentially, with the order of injection changing daily: thus, the time between injection in the first and the last rats in a group varied by a few minutes. In the ethanol training sessions, two auditory stimuli were used, i.e., a tone (A) and a buzzer (B). Stimuli A and B lasted 15 min, and started respectively at two different times during the time course of ethanol action: maximum effect, beginning with the drug injection (called "state +") and evanescence, beginning with the return of body temperature close to pre-injection levels (called "state -"). These states were previously identified in a pilot study of drug-induced hypothermia. Stimulus A was presented 35 min after drug injection, when ethanol reached its maximum effect. Stimulus B was presented 170 min after drug injection, when body temperature was close to that before injection. After presentation of stimulus $\mathrm{B}$, the rats remained in their cages for an additional 10 min until the session was completed. During the saline training session, stimuli C (bip) and D (white noise) were presented at times equivalent to those for stimuli $\mathrm{A}$ and $\mathrm{B}$ during the ethanol training sessions, respectively. "State +" stimuli (A and C for the ethanol and saline cues, respectively) and "state -" stimuli (B and D for the ethanol and saline 
cues, respectively) were counterbalanced. Following these 20 training sessions, the test sessions were run.

Tests. The rats were submitted to four tests. Each test was run over 2 days. Refresher sessions (one ethanol session and one saline session, identical to the last training session) were included between each double session of tests. Injections (ethanol or saline) were given $20 \mathrm{~min}$ after transferral of the rats to the experimental room. The ethanol dose used in the test was the same as that used during the last ethanol training session for each respective group.

On the first day of test 1, the rats were injected with ethanol. Stimulus C (saline training session) was presented 35 min after ethanol injection and was of the same duration as that presented during the training session (15 min). Stimulus B was presented 170 min after ethanol injection (as in the training sessions). On the second day of test 1 , the rats were injected with saline. Stimulus A ("state +" during ethanol training session) was presented $35 \mathrm{~min}$ after the saline injection and had the same duration as in the training session (15 min). Stimulus D was presented 170 min after the saline injection (as in the training sessions).

On the first day of test 2 , the rats were injected with ethanol. Stimulus A was presented both 35 and 170 min after ethanol injection, having the same duration as in the training session (15 min). The second day of test 2 differed from the first day in the presentation of stimulus B for $15 \mathrm{~min}$ at the two times when stimulus A was presented.

Tests 3 and 4 were similar to tests 1 and 2 , respectively, but with changes in the duration and period of presentation of the stimuli: the presentation of the stimuli was anticipated by 30 min relative to tests 1 and 2 , and their duration was extended to $45 \mathrm{~min}$. These anticipated and extended stimuli did not follow the same schedule as used in the training sessions (standard presentation). They were presented under the effect of a substance (ethanol or saline) with which they were not associated during the training phase (e.g., stimuli $\mathrm{A}$ and $\mathrm{C}$ for test 3 ). They were also out of context regarding their own state since they were presented at a time differing from the standard presentation used during the training sessions (e.g., stimuli A and B for test 4). Table 1 shows the design of both the training and test session procedures.

Data analysis. Body temperature data were collected every minute, and are presented as the mean differences between consecutive 5-min blocks during the time course after injection. Changes in temperature within a block were calculated by subtracting the temperature value obtained in the block im-

Table 1. Experimental design of the training and test session procedures.

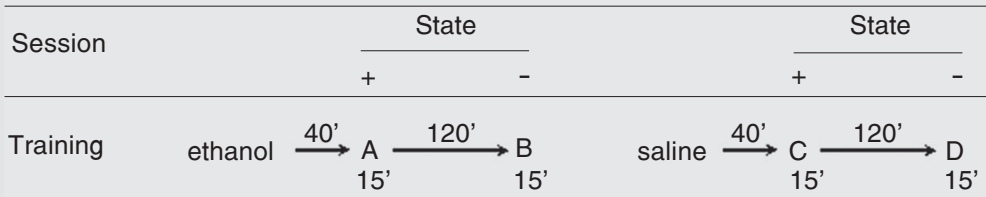

Test

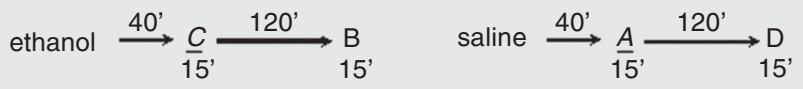

Test 2

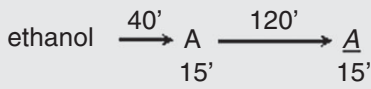

$$
\text { ethanol } \stackrel{40^{\prime}}{\longrightarrow} \frac{B}{15}, \stackrel{120^{\prime}}{\longrightarrow} \text { B } 15^{\prime}
$$

Test 3

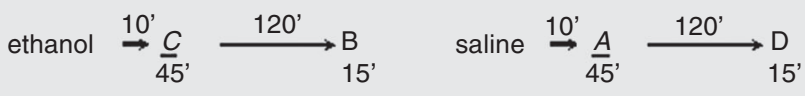

Test 4

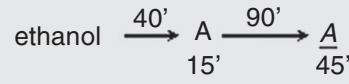

$$
\text { ethanol } \stackrel{10^{\prime}}{\rightarrow} \frac{B}{45^{\prime}}, \stackrel{120^{\prime}}{\longrightarrow} \text { B } 15^{\prime}
$$

Ethanol and saline injections were given over a period of approximately 5 min. State: + = maximum effect after drug injection; - = return of body temperature to pre-injection levels. Auditory stimuli ( 15 or $45 \mathrm{~min}$ ): $\mathrm{A}=$ tone; $\mathrm{B}=$ buzzer; $\mathrm{C}=$ bip; $\mathrm{D}=$ white noise. Underlined italic letters indicate altered stimuli relative to the training session. 
mediately before injection (pre-injection block) from those obtained in the others. These data were subjected to three-way repeated measures ANOVA (analysis of variance) using groups, sessions and blocks as main factors. Post hoc Newman-Keuls comparisons were performed to determine where differences among blocks existed in the sessions. The level of significance was set at $\mathrm{P}$ $\leq 0.05$ in all statistical analyses.

During the course of the test sessions, some animals from the ID group died. The doses and the increasing doses of ethanol used in the present study are not considered to be lethal (see Ref. 26). However, a cumulative toxic effect of ethanol may have occurred in the animals who died. The data for these subjects were discarded, with 8 subjects remaining in each group.

\section{Results}

Figure 1. Time course after injection of mean body temperature differences for $\mathrm{CD}(\mathrm{N}=8)$ and ID $(\mathrm{N}=8)$ groups during the first and tenth training sessions (shown as 5-min blocks). The period of exteroceptive stimulus presentation is indicated between parallel bars. Stimuli $A$ and $\mathrm{B}$ were presented during blocks 8 to 10 , and 35 to 37 , respectively, during the ethanol training session (left panel). Stimuli $C$ and $D$ were presented during blocks 8 to 10 , and 35 to 37 , respectively, during the saline training session (right panel). Ethanol was injected at a dose of $1.4 \mathrm{~g} / \mathrm{kg}$ in the CD group on both days in the ethanol training session, and at doses of 1.2 and $2.0 \mathrm{~g} / \mathrm{kg}$ during the first and tenth ethanol training sessions, respectively, in the ID group. Statistical differences for blocks in relation to the blocks before injection were indicated with small bars and statistical differences between sessions were indicated with asterisks (ANOVA and post hoc Newman-Keuls test). $\mathrm{CD}=$ constant dose; ID = increasing dose.

\section{Baseline data}

$\mathrm{CD}(\mathrm{N}=8)$ and ID $(\mathrm{N}=8)$ groups showed no significant differences during the last baseline session $(\mathrm{F}(1,14)=0.006 ; \mathrm{P}=0.94)$. The results revealed stable body temperatures during the session in which no injection was given (data not shown).

\section{Training sessions}

Figure 1 shows the mean differences in body temperature for the first and tenth training sessions. For the first ethanol training session, ANOVA revealed an effect of blocks $(\mathrm{F}(38,532)=46.17 ; \mathrm{P}<0.001)$, indicating that blocks differed significantly from the block immediately before injection for both CD and ID groups. Newman-Keuls tests showed that these statistical differences continued to persist over the time course of ethanol action until block 34 for both groups, when body temperature reached values close to that obtained in the block before injection. As expected, ethanol induced a hypothermic effect immediately after application, with temperature showing a tendency to return to baseline about $3 \mathrm{~h}$ after ethanol injection during the first session, independently of the group.

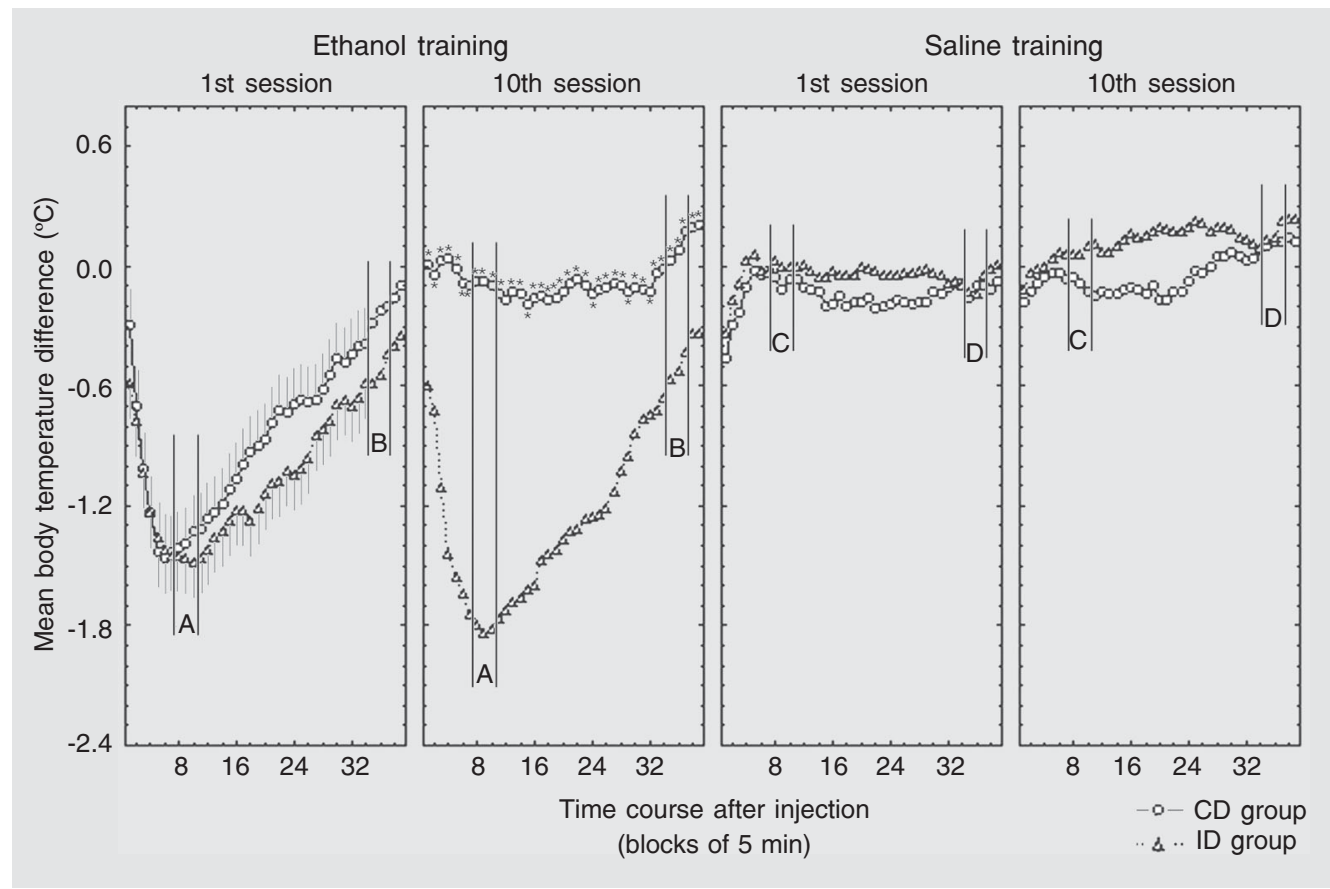


ANOVA showed no differences between the first and tenth ethanol training sessions, indicating that the hypothermic effect was maintained in the ID group during these training sessions $(\mathrm{F}(38,532)=0.95 ; \mathrm{P}=$ 0.54). However, the $\mathrm{CD}$ group exhibited a decrease in mean body temperature difference between the first and tenth ethanol training sessions, revealing the development of tolerance to the hypothermic effect of the drug $(\mathrm{F}(38,532)=14.63 ; \mathrm{P}<0.001)$. No differences were found between the first and tenth saline training sessions for either group $(\mathrm{F}(38,532)=0.52 ; \mathrm{P}=0.99)$.

Refresher sessions included between tests showed no significant differences for $\mathrm{CD}$ or ID groups relative to the last ethanol ( $F$ $(114,988)=0.95 ; \mathrm{P}=0.60)$ and saline $(\mathrm{F}$ $(114,988)=0.60 ; \mathrm{P}=0.99)$ training sessions. This reveals that the conditioning pattern established during the training sessions was maintained. Thus, the data of the last training session (tenth training session) were used as parameters for comparison with those of the other test sessions to detect possible statistical differences.

\section{Tests 1 and 3}

Figure 2 shows the first and second days of tests 1 and 3. For the CD group, ANOVA showed an interaction effect between sessions and blocks on the first day of test 3 in comparison to the tenth ethanol training session $(\mathrm{F}(38,532)=1.67 ; \mathrm{P}=0.008)$. There was a reappearance of the hypothermic effect of the drug in blocks 3 to 22, as indicated by the Newman-Keuls test, compared to the tenth ethanol training session with the anticipated and extended presentation of stimulus $\mathrm{C}$ (associated with saline). This indicates a reduction of the ethanol tolerance effect. No differences were found in the CD group for the first day of test $1(\mathrm{~F}(38,532)=1.06 ; \mathrm{P}$ $=0.37)$ or for both days of test $3(\mathrm{~F}(76,798)$ $=0.65 ; \mathrm{P}=0.99$ ).

The ID group displayed differences for both days of tests 1 and 3. On the first day of test 1 , statistical differences were observed in blocks 12 to 19 compared to the tenth

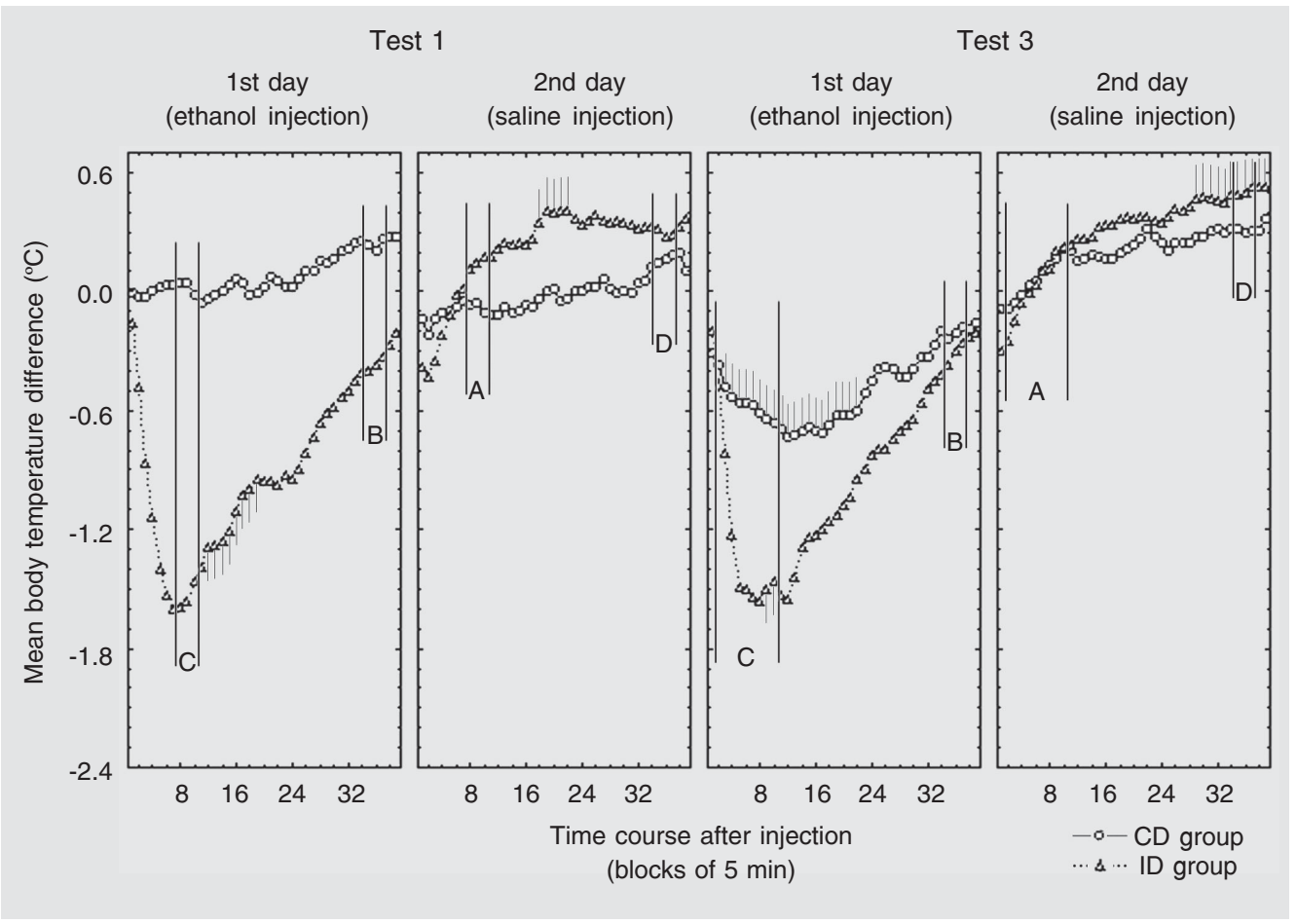

Figure 2. Mean body temperature differences over the time course after injection (shown as 5-min blocks) for tests 1 and 3 in CD $(\mathrm{N}=8)$ and ID $(\mathrm{N}=8)$ groups. The period of exteroceptive stimulus presentation is indicated between parallel bars. On the first day of tests 1 and 3 , ethanol was injected at the same dose as that used during the tenth ethanol training session for the respective groups, and stimuli $C$ and $B$ were presented. On the second day of tests 1 and 3 , saline was injected and stimuli $A$ and $D$ were presented. Statistical differences for sessions and blocks in relation to the last training session are indicated by small bars (ANOVA and post hoc Newman-Keuls test). $\mathrm{CD}=$ constant dose; ID = increasing dose. 
ethanol training session $(\mathrm{F}(38,532)=37.20$; $\mathrm{P}<0.001)$. This indicates a localized effect of the hypothermia reduction produced by the standard presentation of stimulus $\mathrm{C}$ (associated with saline). On the first day of test 3 , the anticipated and extended presentation of stimulus $\mathrm{C}$ produced similar results. However, these results were less evident than those obtained with the standard presentation of stimulus $\mathrm{C}$ on the first day of test 1 . Significant differences compared to the tenth ethanol training session were found in blocks 9 and $10(\mathrm{~F}(38,532)=36.70 ; \mathrm{P}<0.001)$ and, as indicated by the Newman-Keuls test, marginal differences occurred in blocks 14 to 16.

In comparison to the tenth saline training session, the second day of test 1 showed an interaction effect between sessions and blocks for the ID group $(\mathrm{F}(38,532)=4.06 ; \mathrm{P}$ $<0.001)$. There was a tendency to an increase in body temperature with the standard presentation of stimulus A (associated with drug "state +"), observed in blocks 18 to 22 . On the second day of test 3 , ANOVA indi- cated that this hyperthermia was more evident with the anticipated and extended presentation of stimulus A $(\mathrm{F}(38,532)=4.93$; $\mathrm{P}$ $<0.001)$. In this case, Newman-Keuls tests showed that differences occurred from block 29 onwards.

\section{Tests 2 and 4}

Figure 3 shows the first and second days of tests 2 and 4 . For the CD group, ANOVA indicated an interaction effect between sessions and blocks on the second day of both tests 2 and $4(\mathrm{~F}(76,798)=1.57 ; \mathrm{P}=0.01)$ compared to the tenth ethanol training session. Newman-Keuls tests showed a small decrease in body temperature from blocks 20 to 23 for test 2 (standard presentation of stimulus B), and from blocks 20 to 25 for test 4 (anticipated and extended presentation of stimulus B). This hypothermia indicates a reduction in the ethanol tolerance effect for the $\mathrm{CD}$ group with the presentation of stimulus B (associated with drug "state -"). However, the CD group showed no differences
Figure 3. Mean body temperature differences over the time course after injection (shown as 5 -min blocks) for tests 2 and 4 in CD $(\mathrm{N}=8)$ and ID $(\mathrm{N}=8)$ groups. An ethanol injection was given at the same dose as that used during the tenth ethanol training session for the respective groups. The period of exteroceptive stimulus presentation is indicated between parallel bars. Stimulus A was presented on the first day and stimulus B on the second day. Statistical differences for sessions and blocks in relation to the last training session are indicated by small bars (ANOVA and post hoc Newman-Keuls test). $\mathrm{CD}=$ constant dose; ID = increasing dose.

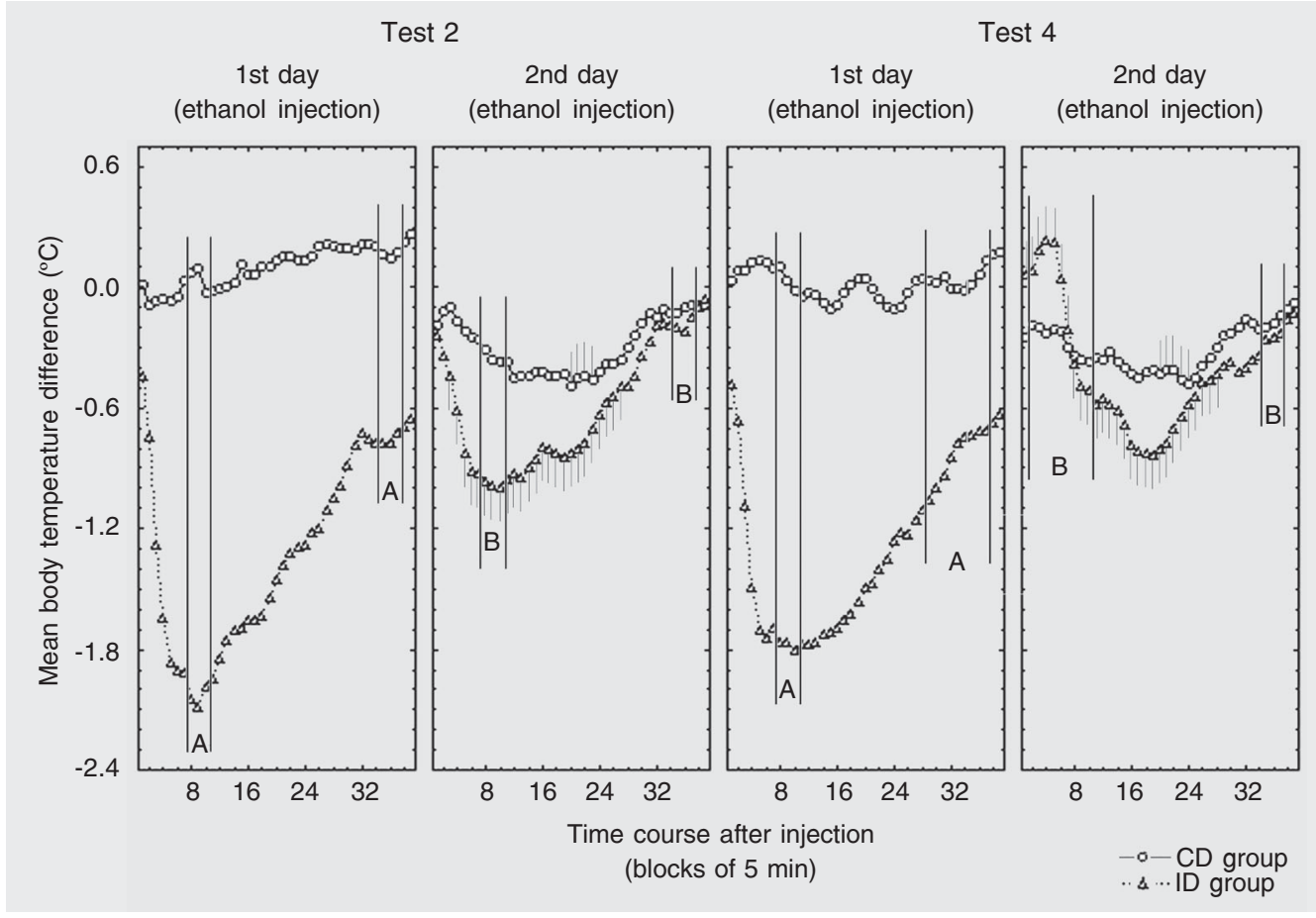


for the first day of both tests 2 and 4 $(\mathrm{F}(76,798)=0.75 ; \mathrm{P}=0.93)$.

The ID group also showed no differences for the first day of tests 2 and $4(\mathrm{~F}(76,722)=$ 0.49 ; $\mathrm{P}=0.99)$. However, ANOVA indicated statistical differences between sessions and blocks for the ID group on the second day of both tests compared to the tenth ethanol training session $(\mathrm{F}(76,722)=5.99 ; \mathrm{P}<$ 0.001 ). On the second day of test 2 , the hypothermic effect was reduced with the standard presentation of stimulus $B$, with differences being found for blocks 3 to 27 . On the second day of test 4 , the anticipated and extended presentation of stimulus B altered the time course of the ethanol effect after ethanol injection for the ID group, with differences being found for blocks 1 to 28 compared to the tenth ethanol training session. The hypothermic effect of ethanol did not appear immediately after injection, as seen during the ethanol training session.

\section{Discussion}

An ethanol but not a saline injection produced a hypothermic effect in both $\mathrm{CD}$ and ID groups, confirming previous reports (e.g., Ref. 13).

CD animals developed tolerance to the hypothermic effect of ethanol from the fourth ethanol training session on (data not shown). This confirms the findings of Mansfield and Cunningham (2) who obtained tolerance to the hypothermic effect of ethanol using a dose of $1.4 \mathrm{~g} / \mathrm{kg}$, also from the fourth session onwards (employing a volume of $8.86 \mathrm{~mL} /$ $\mathrm{kg}$ instead of $5.0 \mathrm{~mL} / \mathrm{kg}$ as used in the present study). The maximum intensity of ethanol effect was about $1.5^{\circ} \mathrm{C}$ in both studies; however, this maximum was recorded 35 min after ethanol injection in the current experiment and after $60 \mathrm{~min}$ in the study by Mansfield and Cunningham (2).

However, the ID group did not show a reduction in the hypothermic effect of ethanol during the same sequence of sessions, indicating that the experimental procedure of progressive dose changes during the training sessions was sufficient to maintain this hypothermic effect. This sustained hypothermic effect does not necessarily mean that this group was not undergoing a tolerance process, but rather that the reduction in the hypothermic effect was counteracted by the increased ethanol dose.

The time course of the hypothermic effect of ethanol indicates that two different internal states exist during the action of ethanol. These internal states were designated as "state +" and "state -" and respectively represent the moments of maximum effect of ethanol and its evanescence in the rats. This process was clearly observed by the hypothermic effect of the drug in the first ethanol training session in both CD and ID groups. The disappearance of this ethanol effect across the successive training sessions demonstrates the effect of drug tolerance in the $\mathrm{CD}$ group. However, this disappearance of the hypothermic effect of ethanol is an effect of drug tolerance and cannot be confused with a saline effect. Likewise, for the ID group, the evanescent effect of ethanol, considered as "state -", is a moment of reduction in the hypothermic effect of ethanol after its maximum effect, considered as "state +" (14) and not a saline effect or a simple effect of drug tolerance.

The presentation of exteroceptive stimuli associated with the "+" and "--" states during testing produced changes in the body temperatures of the animals in both the CD and ID groups. This indicates that the exteroceptive stimuli associated with "+" and "-" states acquired modulatory associative properties during the time course of ethanol action and tolerance. These results do not confirm the suggestion by Revusky and Garcia (27) that "the Pavlovian practice (28) of pairing external stimuli with drug states may be equally inefficient because such stimuli are not readily associable with physiological aftereffects." (p. 31). 
Stimulus A properties (associated with drug "state $+")$

The presentation of stimulus $\mathrm{A}$ in its standard duration - previously associated with drug "state + " in the ethanol training session - induced brief hyperthermia in the ID group upon saline injection (test 1). This hyperthermic effect seen in the ID group was more evident during the anticipated and extended presentation of stimulus A in test 3 than that observed in test 1 . Such hyperthermia can be interpreted as a consequence of the control exerted by stimulus A (drug "state + ") on the compensatory response to the hypothermic effect of ethanol: the hyperthermic component of the compensatory response was present - during the test with saline injection - as a consequence of the properties of stimulus A. Thus, these results support Siegel (7), who suggests the existence of a counteradjustment response towards homeostatic balance, dislocated by drug action. Furthermore, these findings indicate that the exteroceptive cue effects were also associated with the compensatory response.

In the $\mathrm{CD}$ group, stimulus $\mathrm{A}$ did not induce changes in body temperature in any test condition, either with saline (tests 1 and 3 ) or at the time points corresponding to drug "state -" (tests 2 and 4). This indicates that stimulus A does not possess the excitatory component associated with the primary drug effect, since the subjects showed a progressive decrease in the hypothermic effect of ethanol over the training sessions. Stimulus A was associated with ethanol tolerance.

\section{Stimulus B properties (associated with drug "state -")}

The presentation of stimulus B - previously associated with "state -" during the ethanol training sessions - at the time points of drug "state +" reduced the ethanol toler- ance effect in the CD group (tests 2 and 4). This return of the hypothermic effect of ethanol was more intense with the anticipated and extended presentation of the exteroceptive stimulus (test 4) than with the standard presentation (test 2). Stimulus B, regardless of its presentation, either standard (test 2) or anticipated and extended (test 4), promoted a strong reduction in the hypothermic effect of ethanol in the ID group. In this case, control of this stimulus is unrelated to ethanol tolerance, since it is not associated with the development of ethanol tolerance, but rather with the evanescent component of the action of ethanol.

However, the reduction in the tolerance effect observed in the $\mathrm{CD}$ group can also be explained by the second ethanol injection on two successive days. Test 2 shows that with the standard presentation of stimulus $\mathrm{B}$, the body temperature of the animals was already decreasing before the exteroceptive stimulus was presented. Thus, the novel condition, represented by the second successive ethanol injection, may have been sufficient to rupture the tolerance established in the CD group. This hypothesis suggests that the animals in this group would have learned the alternate ethanol-saline training session sequences used in the experiment.

The explanation concerning the second ethanol injection on two successive days can also be used for the ID group. In this case, the decrease in body temperature during test 2 was lower than that seen during the tenth ethanol training session. This reduction in intensity of the hypothermic effect may be understood as a consequence of the development of drug tolerance. However, it does not explain the findings of test 4 . In this test, the presentation of stimulus B with an extended duration, $5 \mathrm{~min}$ after drug injection (initial period of "state +"), retarded the immediate effect of ethanol in the ID group: there was an initial increase in the body temperature of the animals upon presentation of the exteroceptive stimulus, although this increase did not 
persist during the delayed effect of ethanol.

Stimulus B, presented during "state +" of the ethanol time course, produced an ambiguous condition in the ID group, because stimulus B indicates an evanescence period of the effect of ethanol when the rat is under the primary effect of this drug. This may represent a conflicting situation in which the interoceptive and exteroceptive stimuli signal opposite or different responses (29). Two different components occurred during the beginning of the exteroceptive stimulus presentation: the hypothermic effect of ethanol and the control exerted by the B stimulus (drug "state -"). Thus, initially, hyperthermia was present, followed by a hypothermic effect of ethanol, which was delayed or unexpected at that point.

\section{Stimulus C properties (associated with saline)}

Stimulus C, previously associated with the saline injection during training, when presented in its standard duration, induced no change in the temperature of ethanol-injected animals in the CD group (test 1). This indicates a homeostatic equilibrium in $\mathrm{CD}$ animals at the time when the exteroceptive stimulus was presented (35 min after injection). However, when presentation of stimulus $\mathrm{C}$ was anticipated and extended in the $\mathrm{CD}$ group, it caused a decrease in ethanol tolerance (test 3). The reappearance of the hypothermic effect of ethanol with the anticipated and extended presentation of stimulus $\mathrm{C}$ in the ethanol-injected animals in test 3 reveals the presence of a non-drug effect state controlled by the exteroceptive stimulus. Thus, only the anticipated and extended presentation of stimu- lus $\mathrm{C}$ could override the control of stimulus $\mathrm{A}$ on tolerance in the $\mathrm{CD}$ group established during the training sessions.

Anticipation and extension of stimulus $\mathrm{C}$ (test 3) was a novel condition for the $\mathrm{CD}$ group that might have affected drug tolerance (10). Thus, presentation of an exteroceptive stimulus in this new condition may have been responsible for attenuating the compensatory effect, and consequently, the tolerance established during the training sessions in the $\mathrm{CD}$ group.

Usually, the presentation of stimulus $\mathrm{C}$ in its standard duration to the ID group (test 1) induced a local attenuation effect on hypothermia. The anticipated and extended presentation of stimulus $\mathrm{C}$ (test 3 ) produced the same changes in body temperature in this group, although less evident. The internal state in the initial part of the hypothermic effect of ethanol may have been more effective than that elicited by stimulus $\mathrm{C}$. This suggests that the neutral property of stimulus $\mathrm{C}$, associated with saline, reduced the effect of ethanol more clearly during its time course when the hypothermic effect of ethanol was still strong (maximum action), but at the beginning of the ethanol evanescence period.

These findings indicate that ethanol tolerance is subjected to control by exteroceptive stimuli. However, to evaluate this effect of context on ethanol tolerance it is necessary to examine the time course of drug action. Moreover, the associative properties of context are different as a function of this time course. Thus, intervention programs may need to consider these effects of context associated with the time course of drug action and drug tolerance.

\section{References}

1. Hinson RE, Siegel S. The contribution of Pavlovian conditioning to ethanol tolerance and dependence. In: Rigter H, Crabbe JC (Editors), Alcohol tolerance and dependence. New York: Elsevier/North-
Holland Biomedical Press; 1980.

2. Mansfield JG, Cunningham CL. Conditioning and extinction of tolerance to the hypothermic effect of ethanol in rats. J Comp Physiol 
Psychol 1980; 94: 962-969.

3. Wenger JR, Tiffany TM, Bombardier C, Nicholls K, Woods SC Ethanol tolerance in the rat is learned. Science 1981; 213: 575-577.

4. Morato GS, Khanna JM. N-methyl-D-aspartate receptors, nitric oxide, and ethanol tolerance. Braz J Med Biol Res 1996; 29: 14151426.

5. Poulos CX, Cappell H. Homeostatic theory of drug tolerance: a general model of physiological adaptation. Psychol Rev 1991; 98 : 390-408.

6. Ramsay DS, Woods SC. Biological consequences of drug administration: implications for acute and chronic tolerance. Psychol Rev 1997; 104: 170-193.

7. Siegel S. A Pavlovian conditioning analysis of morphine tolerance. In: Krasnegor NA (Editor), Behavioral tolerance: research and treatment implications. Rockville: National Institute on Drug Abuse Research Monograph 18; 1978.

8. Siegel S. The role of conditioning in drug tolerance and addiction. In: Keehn JD (Editor), Psychopathology in animals: Research and clinical applications. New York: Academic Press; 1979.

9. Le AD, Poulos CX, Cappell H. Conditioned tolerance to the hypothermic effect of ethyl alcohol. Science 1979; 206: 1109-1110.

10. Siegel S, Sdao-Jarvie K. Attenuation of ethanol tolerance by a novel stimulus. Psychopharmacology 1986; 88: 258-261.

11. Tabakoff B, Melchior CL, Hoffman P. Factors in ethanol tolerance. Science 1984; 224: 523-524.

12. Siegel S. Pharmacological conditioning and drug effects. In: Goudie AJ, Emmett-Oglesby MW (Editors), Psychoactive drugs: Tolerance and sensitization. New Jersey: Humana Press; 1989.

13. Siegel S. Pavlovian conditioning and ethanol tolerance. In: Lindros $\mathrm{KO}$, Ylikahri R, Kiianmaa K (Editors), Advances in biomedical alcohol research. Oxford: Pergamon Press; 1987.

14. Solomon RL, Corbit JD. An opponent-process theory of motivation. I. Temporal dynamics of affect. Psychol Rev 1974; 81: 119-145.

15. Elsmore TF, Manning FJ. Time course and dose-response effects of orally administered delta-9-THC on interval schedule performance of the rat. Life Sci 1974; 15: 481-489.

16. Revusky S, Coombes S. Long-delay associations between drug states produced in rats by injecting two drugs in sequence. $J$ Comp Physiol Psychol 1982; 96: 549-556.

17. Krank MD, Bennett D. Conditioned activity and the interaction of amphetamine experience with morphine's activity effects. Behav Neural Biol 1987; 48: 422-433.

18. Taukulis HK. Conditional hyperthermia in response to atropine associated with a hypothermic drug. Psychopharmacology 1986; 90: 327-331.

19. Siegel S. State dependent learning and morphine tolerance. Behav Neurosci 1988; 102: 228-232.

20. Overton DA. State-dependent or "dissociated" learning produced with pentobarbital. J Comp Physiol Psychol 1964; 57: 3-12.

21. Overton DA. Contextual stimulus effects of drugs and internal states. In: Balsam PD, Tomie A (Editors), Context and learning. New Jersey: Lawrence Erlbaum Associates; 1985.

22. Cunningham $\mathrm{CL}$. Alcohol as a cue for extinction: State dependency produced by conditioned inhibition. Anim Learn Behav 1979; 7: 4552.

23. Nakagawa $\mathrm{Y}$, Iwasaki T. Ethanol-induced state-dependent learning is mediated by 5 -hydroxytryptamine 3 receptors but not by $\mathrm{N}$-methylD-aspartate receptor complex. Brain Res 1995; 686: 70-76.

24. Bindra D, Palfai T. Nature of positive and negative incentive-motivational effects on general activity. J Comp Physiol Psychol 1967; 63: 288-297.

25. Ades $\mathrm{C}$, Bueno JL. Are conditioned stimuli determinants of oriented exploratory behavior in the rat? Behav Neural Biol 1984; 41: 63-70.

26. Crowell $C R$, Hinson RE, Siegel $S$. The role of conditional drug responses in tolerance to the hypothermic effects of ethanol. Psychopharmacology 1981; 73: 51-54.

27. Revusky S, Garcia J. Learned associations over long delays. In: Bower GH (Editor), The psychology of learning and motivation: advances in research and theory. New York: Academic Press; 1970.

28. Bykov KM. The cerebral cortex and the internal organs. Moscow: Foreign Language Publishing; 1957.

29. Jarbe TU, Johansson B. Interaction between drug discriminative stimuli and exteroceptive, sensory signals. Behav Neurosci 1984; 98: 686-694. 